\title{
Spontaneous symmetry breaking of Bose-Fermi mixtures in double-well potentials
}

\author{
S. K. Adhikari, ${ }^{1, *}$ B. A. Malomed ${ }^{2, \dagger}$ L. Salasnich,,${ }^{3, \dagger}$ and F. Toigo ${ }^{3, \S}$ \\ ${ }^{1}$ Instituto de Física Teórica, Universidade Estadual Paulista, 01.140-070 São Paulo, São Paulo, Brazil \\ ${ }^{2}$ Department of Physical Electronics, School of Electrical Engineering, Faculty of Engineering, Tel Aviv University, Tel Aviv 69978, Israel \\ ${ }^{3}$ Consiglio Nazionale delle Ricerche and Consorzio Nazionale Interuniversitario per le Scienze Fisiche della Materia, Unità di Padova, \\ Dipartimento di Fisica “Galileo Galilei," Università di Padova, Via Marzolo 8, I-35131 Padova, Italy
}

(Received 11 December 2009; published 26 May 2010)

\begin{abstract}
We study the spontaneous symmetry breaking (SSB) of a superfluid Bose-Fermi (BF) mixture in a double-well potential (DWP). The mixture is described by the Gross-Pitaevskii equation (GPE) for the bosons, coupled to an equation for the order parameter of the Fermi superfluid, which is derived from the respective density functional in the unitarity limit (a similar model applies to the BCS regime, too). Straightforward SSB in the degenerate Fermi gas loaded into a DWP is impossible, as it requires an attractive self-interaction, and the intrinsic nonlinearity in the Fermi gas is repulsive. Nonetheless, we demonstrate that the symmetry breaking is possible in the mixture with attraction between fermions and bosons, like ${ }^{40} \mathrm{~K}$ and ${ }^{87} \mathrm{Rb}$. Numerical results are represented by dependencies of asymmetry parameters for both components on particle numbers of the mixture, $N_{\mathrm{F}}$ and $N_{\mathrm{B}}$, and by phase diagrams in the $\left(N_{\mathrm{F}}, N_{\mathrm{B}}\right)$ plane, which displays regions of symmetric and asymmetric ground states. The dynamical picture of the SSB, induced by a gradual transformation of the single-well potential into the DWP, is reported too. An analytical approximation is proposed for the case when the GPE for the boson wave function may be treated by means of the Thomas-Fermi (TF) approximation. Under a special linear relationship between $N_{\mathrm{F}}$ and $N_{\mathrm{B}}$, the TF approximation allows us to reduce the model to a single equation for the fermionic function, which includes competing repulsive and attractive nonlinear terms. The latter one directly displays the mechanism of the generation of the effective attraction in the Fermi superfluid, mediated by the bosonic component of the mixture.
\end{abstract}

DOI: 10.1103/PhysRevA.81.053630

PACS number(s): 03.75.Ss, 03.75.Hh

\section{INTRODUCTION}

The achievement of quantum degeneracy in bosonic [1] and fermionic [2] gases of alkali metal atoms has opened the way to the investigation and manipulation of novel states of atomic matter, such as Bose-Einstein condensates (BEC) [3,4] and superfluid Fermi gases [5]. A simple but reliable theoretical tool for the study of these trapped degenerate gases is the density-functional theory [6]. In particular, the Gross-Pitaevskii equation (GPE), which accurately describes BECs in dilute gases, is the Euler-Lagrange equation produced by the Thomas-Fermi (TF) density functional which takes into account the inhomogeneity of the condensate [4]. In parallel, many properties of superfluid Fermi gases with balanced (equally populated) spin components, and the formation of various patterns in them, can be accurately described, under the conditions of the BCS-BEC crossover, by an extended TF density functional and its time-dependent version, as has been shown recently [7-10].

One of the fundamental effects in nonlinear media, including BEC, which has been studied in detail, is the spontaneous symmetry breaking (SSB) in double-well potentials (DWPs). Asymmetric states trapped in symmetric DWPs are generated by symmetry-breaking bifurcations from obvious symmetric or antisymmetric states, in the media with the attractive or repulsive intrinsic nonlinearity, respectively [11]. [The SSB under the action of competing attractive (cubic) and repulsive

\footnotetext{
*adhikari@ift.unesp.br

${ }^{\dagger}$ malomed@post.tau.ac.il

${ }^{\ddagger}$ salasnich@pd.infn.it

§lavio.toigo@pd.infn.it
}

(quintic) terms was studied, too [12]]. In terms of BEC and other macroscopic quantum systems, the SSB may be realized as a quantum phase transition, which replaces the original symmetric ground state by a new asymmetric one, when the strength of the self-attractive nonlinearity exceeds a certain critical value. Actually, a transition of this type was predicted earlier in the classical context, namely in a model of dual-core nonlinear optical fibers with the self-focusing Kerr nonlinearity [13]. Still earlier, the SSB of nonlinear states was studied, in an abstract form, in the context of the nonlinear Schrödinger equation (NLSE) with a potential term [14], as well in the discrete self-trapping model [15]. The latter approach to the description of the SSB effects was later developed in many works in the form of the two-mode expansion, with each mode representing a mode trapped in one of the potential wells (see Refs. [16] and references therein). As concerns the interpretation of the SSB as the phase transition, it may be categorized as belonging to the first or second kind (known as the sub- or supercritical SSB modes), depending on the form of the nonlinearity, spatial dimension, and the presence or absence of a periodic external potential (an optical lattice) acting along the additional spatial dimension (if any) [17]. In the experiment, the self-trapping of asymmetric states has been demonstrated in the condensate of ${ }^{87} \mathrm{Rb}$ atoms with repulsive interactions [18].

Theoretical studies of the SSB in BECs were extended in various directions. In particular, the symmetry breaking of matter-wave solitons was predicted in various twodimensional (2D) DWP settings [17], including the spontaneous breaking of the skew symmetry of solitons and solitary vortices trapped in double-layer condensates with mutually perpendicular orientations of quasi-one-dimensional optical 
lattices induced in the two layers [19]. A different variety of the two-dimensional (2D) geometry, which gives rise to a specific mode of the SSB, is based on a symmetric set of four potential wells [20] (a three-well system was considered, too [21]). Recently, self-trapping of asymmetric states was predicted in the model of the BEC of dipolar atoms, which interact via long-range forces [22]. SSB was also studied in the context of the NLSE with a general nonlinearity [23]. The symmetry breaking is possible not only in linear potentials composed of two wells but also in a similarly structured pseudopotential, which is produced by a symmetric spatial modulation of the nonlinearity coefficient with two sharp maxima [24].

Another generalization is the study of the SSB in two- [25] and three-component (spinor) [26] BEC mixtures, where the asymmetry of the density profiles in the two wells is coupled to a difference in distributions of the different species. Further, the analysis was extended to a Bose-Fermi (BF) mixture in Ref. [27], where a "frozen" fermion component was treated as a source of an additional potential for bosons. Dynamical manifestations of the symmetry breaking (Josephson oscillations) in a Fermi superfluid trapped in the DWP were recently considered, too [28].

In spite of many realizations of the SSB studied in the models of degenerate quantum gases, the self-trapping of stationary asymmetric states has not yet been considered in fermionic systems. An obvious problem is that a Fermi gas, loaded into a DWP, cannot feature a direct self-attractive nonlinearity, which is necessary to induce the SSB in symmetric states.

The objective of the present work is to introduce a model in which the SSB in a trapped Fermi superfluid is possible due to an effective attraction mediated by a bosonic component mixed with the fermionic one. Actually, we consider the SSB in semitrapped BF mixtures, with the DWP acting on a single species, either the fermionic or bosonic one, as this setting may be sufficient to hold the entire mixture in the trapping potential and induce the SSB in its fermionic component. The analysis is performed in the framework of a mean-field model, which couples, via nonlinear attraction terms, the GPE for the bosonic component to an equation for the fermionic order parameter, derived from the respective density functional. Inducing an effective boson-mediated attraction between the fermions requires an attractive Bose-Fermi (BF) interaction. For this purpose, we take well-known physical parameters corresponding to the ${ }^{87} \mathrm{Rb}$ and ${ }^{40} \mathrm{~K}$ mixture, which features repulsion between rubidium atoms and attraction between the rubidium and potassium, characterized by the respective positive and negative scattering lengths, $a_{\mathrm{B}}>0$ and $a_{\mathrm{BF}}<0$ [29]. We consider the case when the spin-balanced fermionic component of the mixture is in the unitary regime, corresponding to a diverging scattering length which accounts for the interaction between the fermionic atoms with opposite orientations of the spin, $a_{\mathrm{F}} \rightarrow \pm \infty$ (while the BCS regime corresponds to the vanishingly weak attraction, with $a_{\mathrm{F}} \rightarrow-0$; in either limit, the effective fermionic Lagrangian does not depend explicitly on $a_{\mathrm{F}}$ ). In fact, the same model with a different coefficient of the effective self-repulsion in the Fermi superfluid applies to the description of the BF mixture with the fermionic component falling into the BCS regime. Although the self-interaction, induced by the quantum pressure, in the equation for the fermionic order parameter is always repulsive, we demonstrate that the SSB in the fermionic component is indeed possible in the ${ }^{87} \mathrm{Rb}$ and ${ }^{40} \mathrm{~K}$ mixture, due to the $\mathrm{BF}$ attraction which, as stated previously, mediates an effective attraction force in the Fermi superfluid. We also conclude that the attraction can induce symmetry-preserving or symmetry-breaking localization of both components in the semitrapped mixture, depending on the numbers of the bosons and fermions in it.

The article is organized as follows. The model is formulated, in a sufficiently detailed form, in Sec. II. Results produced by the numerical analysis are reported in Sec. III for two variants of the model, with the DWP acting only on either the fermions or the bosons. In Sec. IV, we report approximate analytical results, obtained by means of the TF approximation applied to the GPE for the bosonic wave function. In particular, assuming a specific linear relation between the fermion and boson numbers, we can reduce the model to a single equation for the fermionic wave function with competing self-repulsive and self-attractive terms, the latter one explicitly demonstrating the mechanism of the effective attraction between the fermions mediated by "enslaved" bosons. The analytical results offer a qualitative explanation to general findings produced by the numerical analysis. The article is concluded in Sec. V.

\section{THE MODEL}

Our starting point is a model for the degenerate rarefied quantum gas composed of $N_{\mathrm{B}}$ condensed bosons of mass $m_{\mathrm{B}}$ and $N_{\mathrm{F}}$ fermions of mass $m_{\mathrm{F}}$, in two equally populated spin components at zero temperature. The fermionic component is assumed to be in the superfluid state at unitarity or, alternatively, in the BCS regime. The system is made effectively one-dimensional (1D), assuming that the gas is confined in transverse directions by a tight axisymmetric harmonic potential, with trapping frequencies $\omega_{\perp \mathrm{B}}, \omega_{\perp \mathrm{F}}$ for the bosons and fermions, respectively.

Within the framework of the density-functional theory for superfluids [8,9], the three-dimensional (3D) action of the BF mixture is

$$
S=\int\left(\mathcal{L}_{\mathrm{B}}+\mathcal{L}_{\mathrm{F}}+\mathcal{L}_{\mathrm{BF}}\right) d^{3} \mathbf{r} d t,
$$

where $\mathcal{L}_{\mathrm{B}}$ is the ordinary bosonic Lagrangian density,

$$
\begin{aligned}
\mathcal{L}_{\mathrm{B}}= & \frac{i}{2} \hbar\left(\psi_{\mathrm{B}}^{*} \frac{\partial \psi_{\mathrm{B}}}{\partial t}-\psi_{\mathrm{B}} \frac{\partial \psi_{\mathrm{B}}^{*}}{\partial t}\right)-\frac{\hbar^{2}}{2 m_{\mathrm{B}}}\left|\nabla \psi_{\mathrm{B}}\right|^{2} \\
& -U_{\mathrm{B}}\left|\psi_{\mathrm{B}}\right|^{2}-\frac{2 \pi \hbar^{2} a_{\mathrm{B}}}{m_{\mathrm{B}}}\left|\psi_{\mathrm{B}}\right|^{4},
\end{aligned}
$$

$\psi_{\mathrm{B}}(\mathbf{r}, t)$ is the macroscopic BEC wave function, and the confining potential for the bosons is

$$
U_{\mathrm{B}}(\mathbf{r})=\frac{1}{2} m_{\mathrm{B}} \omega_{\perp \mathrm{B}}^{2} R^{2}+V_{\mathrm{B}}(z),
$$

with $R$ as the transverse cylindric radial coordinate and $V_{\mathrm{B}}(z)$ as the potential acting in the axial direction $z$. The bosonic superfluid velocity is $\mathbf{v}_{\mathrm{B}}(\mathbf{r}, t)=\left(\hbar / m_{\mathrm{B}}\right) \nabla \theta_{\mathrm{B}}(\mathbf{r}, t)$, where $\theta_{\mathrm{B}}(\mathbf{r}, t)$ is the phase of wave function, $\psi_{\mathrm{B}}(\mathbf{r}, t) \equiv \sqrt{n_{\mathrm{B}}(\mathbf{r}, t)} e^{i \theta_{\mathrm{B}}(\mathbf{r}, t)}$, and $n_{\mathrm{B}}(\mathbf{r}, t)$ is the bosonic density. 
The Galilean-invariant Lagrangian density $\mathcal{L}_{\mathrm{F}}$ of the Fermi gas with two equally populated spin components is $[8,30]$

$$
\begin{aligned}
\mathcal{L}_{\mathrm{F}}= & i \frac{\hbar}{4}\left(\psi_{\mathrm{F}} \frac{\partial \psi_{\mathrm{F}}}{\partial t}-\psi_{\mathrm{F}} \frac{\partial \psi_{\mathrm{F}}}{\partial t}\right)-\frac{\hbar^{2}}{8 m_{\mathrm{F}}}\left|\nabla \psi_{\mathrm{F}}\right|^{2} \\
& -\frac{3}{5} \xi \frac{\hbar^{2}}{2 m_{\mathrm{F}}}\left(3 \pi^{2}\right)^{2 / 3}\left|\psi_{\mathrm{F}}\right|^{10 / 3}-U_{\mathrm{F}}\left|\psi_{\mathrm{F}}\right|^{2},
\end{aligned}
$$

where $\psi_{\mathrm{F}}(\mathbf{r}, t)$ is the superfluid order parameter of the Fermi gas at unitary [8], $2 m_{\mathrm{F}}$ is the mass of a pair of fermions with spins up and down, and the potential acting on the fermionic atoms is

$$
U_{\mathrm{F}}(\mathbf{r})=\frac{1}{2} m_{\mathrm{F}} \omega_{\perp \mathrm{F}}^{2} R^{2}+V_{\mathrm{F}}(z)
$$

[cf. its bosonic counterpart (3)]. The fermionic superfluid velocity is $\mathbf{v}_{\mathrm{F}}(\mathbf{r}, t)=\left(\hbar / 2 m_{\mathrm{F}}\right) \nabla \theta_{\mathrm{F}}(\mathbf{r}, t)$, where $\theta_{\mathrm{F}}(\mathbf{r}, t)$ is the phase of the order parameter, $\psi_{\mathrm{F}}(\mathbf{r}, t) \equiv \sqrt{n_{\mathrm{F}}(\mathbf{r}, t)} e^{i \theta_{\mathrm{F}}(\mathbf{r}, t)}$, and $n_{\mathrm{F}}(\mathbf{r}, t)$ is the density of fermionic atoms. Constant $\xi$ in expression (4) is $\xi=1$ in the deep BCS regime, and $\xi \simeq 0.4$ at the unitarity [5]. In calculations reported later, we fixed $\xi=$ 0.45 , assuming the unitarity regime. Lastly, the Lagrangian density $\mathcal{L}_{\mathrm{BF}}$ in expression (1) accounting for the BF interaction is

$$
\mathcal{L}_{\mathrm{BF}}=-\frac{2 \pi \hbar^{2} a_{\mathrm{BF}}}{m_{R}}\left|\psi_{\mathrm{B}}\right|^{2}\left|\psi_{\mathrm{F}}\right|^{2},
$$

where $m_{\mathrm{R}}=m_{\mathrm{B}} m_{\mathrm{F}} /\left(m_{\mathrm{B}}+m_{\mathrm{F}}\right)$ is the respective reduced mass and, as stated previously, $a_{\mathrm{BF}}<0$ corresponds to an attractive $\mathrm{BF}$ interaction, which is necessary to support the SSB of the fermionic component in the presence of the DWP. We stress our assumptions: (i) $a_{\mathrm{BF}}$ is independent of the spin component and (ii) the fermionic density profiles are identical for the two components.

In the nearly $1 \mathrm{D}$ configuration, transverse widths of the atomic distributions are determined by the width of the ground states of the respective harmonic oscillators:

$$
a_{\perp \mathrm{B}}=\sqrt{\hbar /\left(m_{\mathrm{B}} \omega_{\perp \mathrm{B}}\right)}, \quad a_{\perp \mathrm{F}}=\sqrt{\hbar /\left(2 m_{\mathrm{F}} \omega_{\perp \mathrm{F}}\right)},
$$

for the condensed bosons and superfluid fermions at the unitarity. The boson and fermion components exhibit the effective 1D behavior if their chemical potentials are much smaller than the corresponding transverse-trapping energies, $\hbar \omega_{\perp \mathrm{B}}$ and $\hbar \omega_{\perp \mathrm{F}} / 2$. Notice that the effective mass $2 m_{F}$ in the fermionic harmonic length of Eq. (7) is a direct consequence of the coefficient $1 / 8$ in the gradient term $\left|\nabla \psi_{F}\right|^{2}$ of the fermionic Lagrangian (4). This coefficient is chosen to reproduce very accurately the Monte Carlo simulations of the 3D unitary Fermi gas $[8,9,30]$. Under these conditions, we can adopt the known factorized ansatz for the 3D wave functions [31],

$$
\begin{aligned}
& \psi_{\mathrm{B}}(\mathbf{r}, t)=\sqrt{N_{\mathrm{B}}} \frac{e^{-R^{2} /\left(2 a_{\perp \mathrm{B}}^{2}\right)}}{\pi^{1 / 2} a_{\perp \mathrm{B}}} \Phi_{\mathrm{B}}(z, t), \\
& \psi_{\mathrm{F}}(\mathbf{r}, t)=\sqrt{N_{\mathrm{F}}} \frac{e^{-R^{2} /\left(2 a_{\perp \mathrm{F}}^{2}\right)}}{\pi^{1 / 2} a_{\perp \mathrm{F}}} \Phi_{\mathrm{F}}(z, t),
\end{aligned}
$$

where $\Phi_{\mathrm{B}}(z)$ and $\Phi_{\mathrm{F}}(z)$ are the 1D (axial) wave functions, which are subject to the usual normalization conditions,

$$
\int_{-\infty}^{+\infty}\left|\Phi_{\mathrm{B}}(z, t)\right|^{2} d z=\int_{-\infty}^{+\infty}\left|\Phi_{\mathrm{F}}(z, t)\right|^{2} d z=1 .
$$

By inserting expressions (8) and (9) into Eq. (1), we can write the action as $S=S^{(1 \mathrm{D})}-\left(N_{\mathrm{B}} \hbar \omega_{\perp \mathrm{B}}-N_{\mathrm{F}} \hbar \omega_{\perp \mathrm{F}} / 2\right) t$, with the effective $1 \mathrm{D}$ action

$$
S^{(1 \mathrm{D})}=\int d t \int_{-\infty}^{+\infty} d z\left[\mathcal{L}_{\mathrm{B}}^{(1 \mathrm{D})}+\mathcal{L}_{\mathrm{F}}^{(1 \mathrm{D})}+\mathcal{L}_{\mathrm{BF}}^{(1 \mathrm{D})}\right]
$$

where the usual 1D Gross-Pitaevskii Lagrangian density is

$$
\begin{aligned}
\mathcal{L}_{B}^{(1 \mathrm{D})}= & N_{\mathrm{B}}\left[i \frac{\hbar}{2}\left(\Phi_{\mathrm{B}}^{*} \frac{\partial \Phi_{\mathrm{B}}}{\partial t}-\Phi_{\mathrm{B}} \frac{\partial \Phi_{\mathrm{B}}^{*}}{\partial t}\right)-\frac{\hbar^{2}}{2 m_{\mathrm{B}}}\left|\frac{\partial \Phi_{\mathrm{B}}}{\partial z}\right|^{2}\right. \\
& \left.-V_{\mathrm{B}}\left|\Phi_{\mathrm{B}}\right|^{2}-\frac{1}{2} G_{\mathrm{B}}\left|\Phi_{\mathrm{B}}\right|^{4}\right]
\end{aligned}
$$

where the boson self-interaction strength in one dimension is

$$
G_{\mathrm{B}} \equiv 2 N_{\mathrm{B}} \hbar \omega_{\perp \mathrm{B}} a_{\mathrm{B}} .
$$

Further, the 1D fermionic Lagrangian density in Eq. (11) is

$$
\begin{aligned}
\mathcal{L}_{\mathrm{F}}^{(1 \mathrm{D})}= & N_{\mathrm{F}}\left[i \frac{\hbar}{4}\left(\Phi_{\mathrm{F}}^{*} \frac{\partial \Phi_{\mathrm{F}}}{\partial t}-\Phi_{\mathrm{F}} \frac{\partial \Phi_{\mathrm{F}}^{*}}{\partial t}\right)-\frac{\hbar^{2}}{8 m_{\mathrm{F}}}\left|\frac{\partial \Phi_{\mathrm{F}}}{\partial z}\right|^{2}\right. \\
& \left.-\frac{3}{5} A\left|\Phi_{\mathrm{F}}\right|^{10 / 3}-V_{\mathrm{F}}\left|\Phi_{\mathrm{F}}\right|^{2}\right]
\end{aligned}
$$

with the effective strength of the fermionic quantum pressure,

$$
A=\left(3 \pi^{2}\right)^{2 / 3}(3 \xi / 5) \hbar^{2} N_{\mathrm{F}}^{2 / 3} /\left(2 m_{\mathrm{F}} a_{\perp \mathrm{F}}^{4 / 3}\right),
$$

emerging as the coefficient in front of the bulk kinetic energy of the Fermi gas in the unitarity limit. Finally, the 1D Lagrangian density of the BF interaction is

$$
\mathcal{L}_{\mathrm{BF}}^{(1 \mathrm{D})}=-N_{\mathrm{B}} N_{\mathrm{F}} G_{\mathrm{BF}}\left|\Phi_{\mathrm{B}}\right|^{2}\left|\Phi_{\mathrm{F}}\right|^{2},
$$

where the corresponding $1 \mathrm{D}$ interaction strength is

$$
G_{\mathrm{BF}} \equiv 2 \hbar^{2} a_{\mathrm{BF}} /\left(m_{\mathrm{R}} a_{\perp \mathrm{B}} a_{\perp \mathrm{F}}\right) .
$$

For numerical calculations, we set $a_{\perp \mathrm{B}}=a_{\perp \mathrm{F}} \equiv a_{\perp}$ and $\omega_{\perp \mathrm{B}}=\omega_{\perp \mathrm{F}} \equiv \omega_{\perp}$, measuring lengths and time in units of $a_{\perp}$ and $\omega_{\perp}^{-1}$, respectively. This implies that $2 m_{\mathrm{F}}=m_{\mathrm{B}}$ (hence, $m_{\mathrm{R}}=m_{\mathrm{B}} / 3$ ), a condition which is roughly satisfied by the ${ }^{87} \mathrm{Rb}$ and ${ }^{40} \mathrm{~Kb}$ mixture. We consider ${ }^{40} \mathrm{~K}$ atoms in the two equally populated hyperfine states $\left|F=9 / 2, m_{\mathrm{F}}=-9 / 2\right\rangle$ and $\left|F=9 / 2, m_{\mathrm{F}}=-7 / 2\right\rangle$ and ${ }^{87} \mathrm{Rb}$ atoms in the hyperfine state $\left|F=2, m_{\mathrm{F}}=2\right\rangle$. This mixture is a good candidate for experimental study of the SSB because the BF scattering length is negative (as stressed previously), $a_{\mathrm{BF}} \approx-284 a_{0}$, where $a_{0}$ is the Bohr radius [29]. Simultaneously, the scattering length for collisions between rubidium atoms is positive, $a_{\mathrm{B}} \approx 108 a_{0}$ $[3,29,32]$. In principle, the magnetic field inducing the Feshbach resonance can affect the values of $a_{\mathrm{BF}}$ and $a_{\mathrm{B}}$, but we neglect this effect in all the calculations reported in the present work.

The application of the variational procedure to the effective action (11) produces a system of coupled NLSEs,

$$
\left[-\frac{1}{2} \frac{\partial^{2}}{\partial x^{2}}+W_{\mathrm{B}}(x)+g_{\mathrm{B}}\left|\phi_{\mathrm{B}}\right|^{2}+g_{\mathrm{BF}} N_{\mathrm{F}}\left|\phi_{\mathrm{F}}\right|^{2}\right] \phi_{\mathrm{B}}=i \frac{\partial}{\partial \tau} \phi_{\mathrm{B}},
$$

$\left[-\frac{1}{8} \frac{\partial^{2}}{\partial x^{2}}+g_{\mathrm{F}}\left|\phi_{\mathrm{F}}\right|^{4 / 3}+W_{\mathrm{F}}(x)+g_{\mathrm{BF}} N_{\mathrm{B}}\left|\phi_{\mathrm{B}}\right|^{2}\right] \phi_{\mathrm{F}}=\frac{i}{2} \frac{\partial}{\partial \tau} \phi_{\mathrm{F}}$, 
where $x=z / a_{\perp}, \tau=\omega_{\perp} t$, and $\phi_{\mathrm{B}}=a_{\perp}^{1 / 2} \Phi_{\mathrm{B}}, \phi_{\mathrm{F}}=a_{\perp}^{1 / 2} \Phi_{\mathrm{F}}$, $W_{\mathrm{B}}=V_{\mathrm{B}} /\left(\hbar \omega_{\perp}\right), W_{\mathrm{F}}=V_{\mathrm{F}} /\left(\hbar \omega_{\perp}\right)$, and the renormalized interaction coefficients are derived from expressions (13), (15), and (17):

$$
\begin{gathered}
g_{\mathrm{B}}=G_{\mathrm{B}} /\left(\hbar \omega_{\perp} a_{\perp}\right) \equiv 2\left(a_{\mathrm{B}} / a_{\perp}\right) N_{\mathrm{B}}, \\
g_{\mathrm{BF}}=G_{\mathrm{BF}} /\left(\hbar \omega_{\perp} a_{\perp}\right) \equiv 6 a_{\mathrm{BF}} / a_{\perp}, \\
g_{\mathrm{F}}=A /\left(a_{\perp}^{2 / 3} \hbar \omega_{\perp}\right) \equiv\left(3 \pi^{2}\right)^{2 / 3}(3 \xi / 5) N_{\mathrm{F}}^{2 / 3} .
\end{gathered}
$$

Note that the rescaled wave functions are subject to the same normalization conditions as in Eqs. (10), that is,

$$
\int_{-\infty}^{+\infty}\left|\phi_{\mathrm{B}}(x, \tau)\right|^{2} d x=\int_{-\infty}^{+\infty}\left|\phi_{\mathrm{F}}(x, \tau)\right|^{2} d x=1 .
$$

If condition $2 m_{\mathrm{F}}=m_{\mathrm{B}}$ does not hold, the coupled equations can be cast in the same form, with a difference that coefficient $2 m_{\mathrm{F}} / m_{\mathrm{B}}$ appears in front of the second derivative in Eq. (18).

The coupled NLSEs in the form of Eqs. (19) actually generalize the static and dynamical equations for BF systems which were used in various settings in Refs. [33]. In particular, the semiphenomenological equations used for the study of 1D gap solitons in the BF mixture in Ref. [34] are also tantamount to Eqs. (19), up to a difference in coefficients.

To find stationary solutions to Eqs. (18) and (19), we employed a Crank-Nicolson finite-difference scheme for simulations of the equations in imaginary time, using the FORTRAN codes provided in Ref. [35]. We employed space and time steps $\Delta x=0.025$ and $\Delta t=0.001$ and a sufficiently large number of iterations to ensure the convergence. The stability of the stationary solutions against small perturbations was then tested by simulations in real time.

Due to the attractive character of the BF interactions $\left(a_{\mathrm{BF}}<0\right)$, the true ground state of the 3D mixture collapses toward energy $E=-\infty$. Nevertheless, because of the strong transverse confinement, the quasi-1D metastable state has an indefinitely long lifetime [36]. Actually, stationary solutions generated by the imaginary-time integration represent the ground state of the effective 1D BF system based on Eqs. (18) and (19) in the same sense as the famous matter-wave solitons realize the ground state of the quasi-1D condensate of ${ }^{7} \mathrm{Li}$ atoms [37].

\section{RESULTS OF THE NUMERICAL ANALYSIS}

\section{A. Axially trapped fermions and free bosons}

We start the analysis by considering the configuration with the DWP acting solely on the fermionic component:

$$
W_{\mathrm{F}}(x)=\alpha_{\mathrm{F}} x^{2}+\beta_{\mathrm{F}} \exp \left(-\gamma_{\mathrm{F}} x^{2}\right), \quad W_{\mathrm{B}}(z)=0,
$$

where all constants are positive. An elementary consideration of this potential demonstrates that it features the doublewell structure provided that $\alpha_{\mathrm{F}}<\beta_{\mathrm{F}} \gamma_{\mathrm{F}}$, with two symmetric potential minima located at points

$$
x_{\min }= \pm \gamma_{\mathrm{F}}^{-1 / 2} \sqrt{\ln \left(\beta_{\mathrm{F}} \gamma_{\mathrm{F}} / \alpha_{\mathrm{F}}\right)} .
$$

We report results of simulations for $\alpha_{\mathrm{F}}=1 / 2, \beta_{\mathrm{F}}=16$, and $\gamma_{\mathrm{F}}=10$, which adequately represents the generic situation; in this case, Eq. (23) yields $x_{\min } \approx \pm 0.76$.
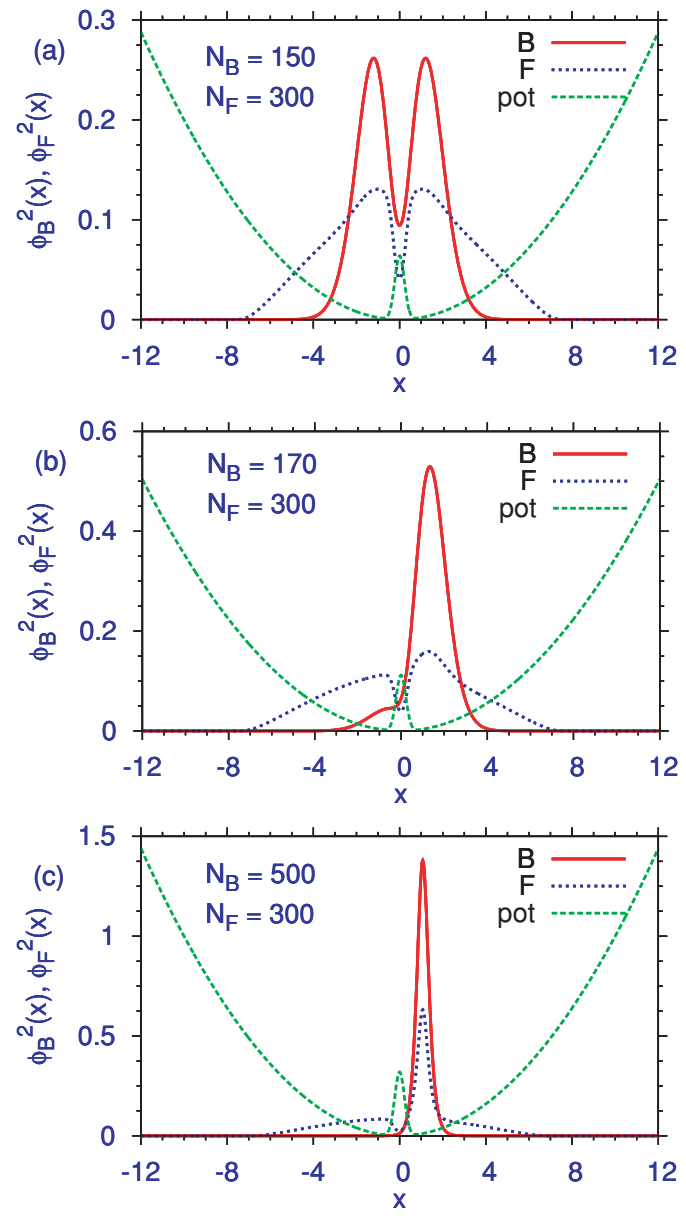

FIG. 1. (Color online) Density profiles of the ${ }^{87} \mathrm{Rb}$ and ${ }^{40} \mathrm{~Kb}$ mixture, $\phi_{\mathrm{B}}^{2}(x)$ and $\phi_{\mathrm{F}}^{2}(x)$, marked by the respective labels, in the case of the DWP (shown in arbitrary units by curve "pot") acting on the fermions, while no axial potential is applied to the bosons. The three panels differ by the number of bosons, $N_{\mathrm{B}}$, as indicated in each panel. Recall that the axial coordinate $x$ is measured in units of the transverse-confinement length, $a_{\perp}$, while $\phi_{\mathrm{B}}^{2}$ and $\phi_{\mathrm{F}}^{2}$ are displayed in units of $a_{\perp}^{-1}$.

The purpose of the analysis is to construct $x$-symmetric and asymmetric ground states of the system, varying the control parameters, and identify the respective SSB bifurcation, that is, the transition to the asymmetric ground state. The asymmetry of its Fermi and Bose components is characterized by parameters

$$
\theta_{\mathrm{F}, \mathrm{B}} \equiv \frac{\int_{0}^{\infty}\left|\phi_{\mathrm{F}, \mathrm{B}}(x)\right|^{2} d x-\int_{-\infty}^{0}\left|\phi_{\mathrm{F}, \mathrm{B}}(x)\right|^{2} d x}{\int_{-\infty}^{+\infty}\left|\phi_{\mathrm{F}, \mathrm{B}}(x)\right|^{2} d x} .
$$

Recall that the denominator in this expression is actually 1 for both species, as per Eq. (21).

In Fig. 1, we display a set of axial (1D) profiles of the densities of both components in the ground state, generated by the integration of Eqs. (18) and (19) in imaginary time, in the case of the BF attraction and weak repulsion between the bosons. The respective values of the interaction coefficients in Eqs. (18) and (19) are taken as per Eqs. (20), with the previously mentioned values of $a_{\mathrm{BF}}$ and $a_{\mathrm{B}}$ for the ${ }^{87} \mathrm{Rb}$ and ${ }^{40} \mathrm{~Kb}$ mixture and a fixed transverse-trapping length, 

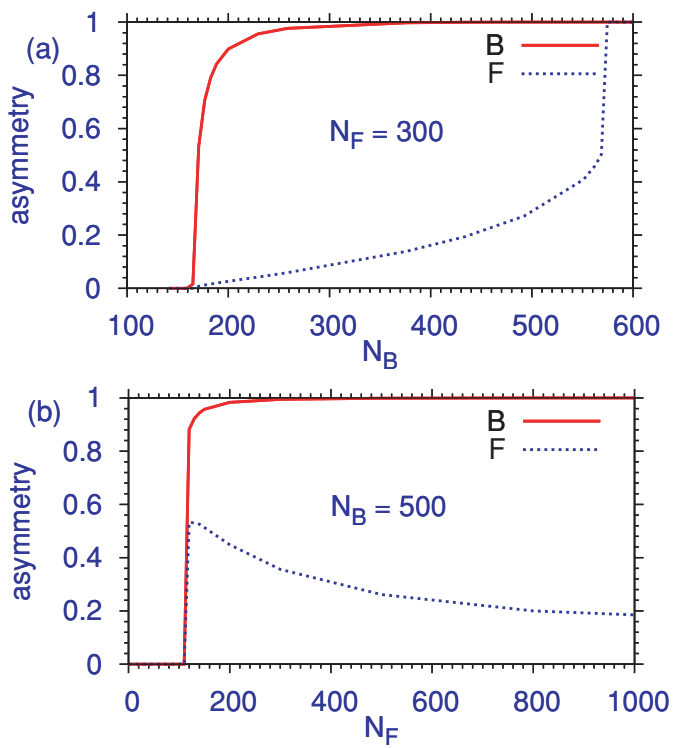

FIG. 2. (Color online) Asymmetry parameters (24), for the bosons (B) and fermions (F) in the ${ }^{87} \mathrm{Rb}$ and ${ }^{40} \mathrm{~Kb}$ mixture, loaded into potential (22), as functions of (a) the number of bosons, $N_{\mathrm{B}}$, at a fixed number of fermions, $N_{\mathrm{F}}=300$, and (b) the number of fermions, at fixed $N_{\mathrm{B}}=500$.

$a_{\perp}=1 \mu \mathrm{m}$, substituting various values of atomic numbers $N_{\mathrm{B}}$ and $N_{\mathrm{F}}$. The figure clearly shows the transition from the symmetric ground state to the asymmetric one, with the increase of the number of bosons, $N_{\mathrm{B}}$, that is, the strength of both the boson-boson and BF interactions. Accordingly, the increase of $N_{\mathrm{B}}$ leads to a stronger overlap between the bosons and fermions and to the SSB (i.e., the transition from symmetric ground states to an asymmetric one), which happens (simultaneously in both components) between $N_{\mathrm{B}}=$ 150 and 170 for a fixed number of fermions, $N_{\mathrm{F}}=300$.

Similar to what is shown in Fig. 1, the symmetric ground state is replaced by an asymmetric one with the increase of $N_{\mathrm{F}}$ at fixed $N_{\mathrm{B}}$, as this implies the strengthening of the BF interaction. The summary of the results for the SSB in the present setting is provided in Fig. 2 by plots of asymmetry parameters (24) versus $N_{\mathrm{B}}$ for fixed $N_{\mathrm{F}}$, and vice versa. Naturally, the SSB of both components happens at the same point [for instance, at $N_{\mathrm{B}} \approx 105$ in panel (a)]. Nevertheless, the resulting bosonic asymmetry is essentially stronger [in Fig. 1(a), up to a point, $N_{\mathrm{B}} \approx 580$, at which both $\theta_{\mathrm{B}}$ and $\theta_{\mathrm{F}}$ attain values very close to 1 ; that is, practically all the atoms are collected in a single potential well]. In Fig. 1(b), the behavior of the fermionic asymmetry, $\Theta_{\mathrm{F}}$, is different: it jumps to a maximum value at the SSB point and then gradually decreases. This difference between the bosonic and fermionic components is natural, as the intrinsic repulsion in the latter one tends to restore the symmetry between the distributions of atoms in the two potential wells.

The results are further summarized in Fig. 3, which displays the phase diagram of the mixture. There are three regions in the $\left(N_{\mathrm{F}}, N_{\mathrm{B}}\right)$ plane: an area where the attraction to fermions cannot keep bosonic atoms in the trapped state ("free bosons"), the region where the bosons and fermions are trapped in the



FIG. 3. (Color online) Phase diagram of the ${ }^{87} \mathrm{Rb}$ and ${ }^{40} \mathrm{~Kb}$ mixture in potential (22), which acts only on the fermions. The diagram shows, on the logarithmic scales in the $\left(N_{\mathrm{B}}, N_{\mathrm{F}}\right)$ plane, regions of the symmetric ground state and of the SSB. "Free bosons" implies delocalization of the bosonic wave function.

symmetric state with respect to the DWP, and the SSB region, where the mixture is trapped in the asymmetric ground state.

An example of the dynamical development of the SSB from an initially symmetric configuration, in the case where the ground state is asymmetric, is presented by Fig. 4. Initially, the bosons and fermions form a stable symmetric bound state, via their mutual attraction, in the single-well potential acting on the fermions, which is taken in the form of (22) with $\beta_{\mathrm{F}}=0$. Then, $\beta_{\mathrm{F}}$ is ramped linearly in time $(0<t<80)$ from $\beta_{\mathrm{F}}=0$ to $\beta_{\mathrm{F}}=16$, which leads to splitting the single potential well into two, as per Eq. (23). The dynamical picture clearly shows the transition of the initial symmetric state into the symmetrybroken one. Both components get spontaneously collected in one of the wells, where they stay together due to the mutual attraction, approaching an equilibrium configuration.

\section{B. Axially trapped bosons and free fermions}

Now, we consider the action of the DWP on the bosons only, taking the potential as

$$
W_{\mathrm{B}}(x)=\alpha_{\mathrm{B}} x^{2}+\beta_{\mathrm{B}} \exp \left(-\gamma_{\mathrm{B}} x^{2}\right), \quad W_{\mathrm{F}}=0 .
$$

For this setting, numerical results are presented with $\alpha_{\mathrm{B}}=1 / 2$, $\beta_{\mathrm{B}}=16$, and $\gamma_{\mathrm{B}}=10$, that is, the same parameters of the DWP as used previously for trapping the fermions. We again aim to
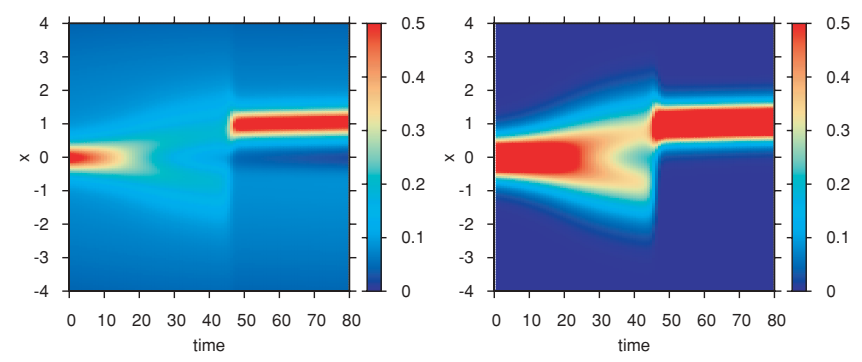

FIG. 4. (Color online) Contour plots for the evolution of densities $\left|\phi_{\mathrm{B}}(x, t)\right|^{2}$ and $\left|\phi_{\mathrm{F}}(x, t)\right|^{2}$ of the (a) bosons and (b) fermions in the ${ }^{87} \mathrm{Rb}$ and ${ }^{40} \mathrm{~Kb}$ mixture. At $t=0$, the fermions are trapped in the single-well potential (22), with $\beta_{\mathrm{F}}=0$. Then, $\beta_{\mathrm{F}}$ linearly increases from 0 to 16 by $t=80$, which implies the transition to the doublewell potential with well-separated symmetric minima. Numbers of particles are $N_{\mathrm{B}}=500$ and $N_{\mathrm{F}}=300$. 




FIG. 5. (Color online) Phase diagram of the ${ }^{87} \mathrm{Rb}$ and ${ }^{40} \mathrm{~K}$ mixture loaded into potential (25) which acts only on the bosons.

construct the ground state of the system as a function of the control parameters and investigate its spontaneous transition into an asymmetric shape.

Results of the analysis for this setting are summarized in the respective phase diagram of the mixture plotted in Fig. 5 (cf. Fig. 3). In this case too, there are three regions in the $\left(N_{\mathrm{F}}, N_{\mathrm{B}}\right)$ plane: an area where the fermions cannot be held in a localized state by the attraction to bosons ("free fermions"), the region where the fermions are trapped, along with the bosons, in a symmetric ground state, and the region where the trapped ground state is asymmetric (SSB) for both the fermionic and bosonic components.

An example of the transition from the symmetric ground state of the BF mixture to an asymmetric one, caused by the increase of the number of fermions from $N_{\mathrm{F}}=10$ to $N_{\mathrm{F}}=$ 1000 while the number of bosons trapped in potential (25) was kept constant, is displayed in Fig. 6. Although the applicability of the functional-density description for $N_{\mathrm{F}}=10$ [in panel (a)] may be disputed, this figure adequately shows the transition to the SSB.
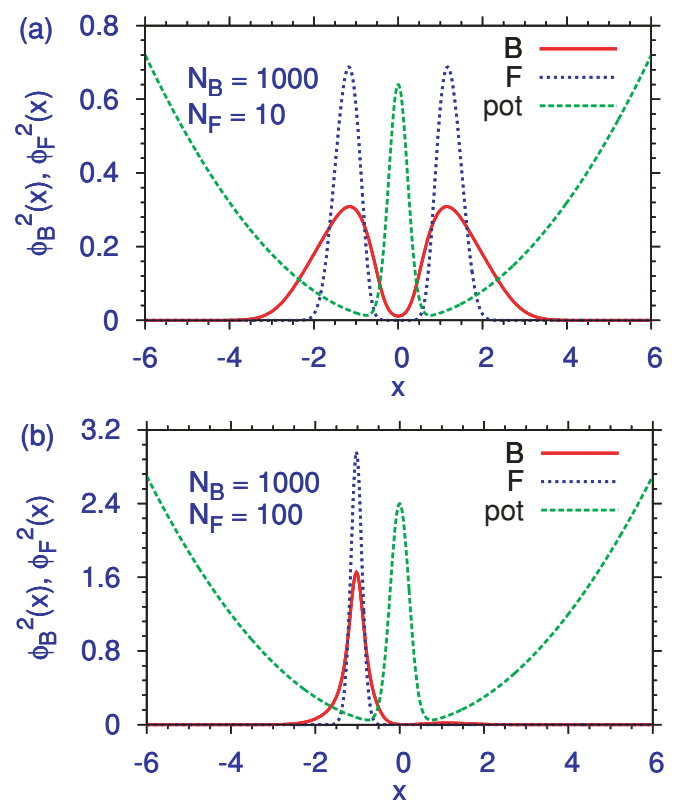

FIG. 6. (Color online) Example of the spontaneous symmetry breaking in the ${ }^{87} \mathrm{Rb}$ and ${ }^{40} \mathrm{~K}$ mixture trapped in potential $(25)$. The two panels differ by the number of fermions: (a) $N_{\mathrm{F}}=10$ and (b) $N_{\mathrm{F}}=1000$.

\section{Case of the fermionic component in the BCS regime}

The analysis presented thus far pertains to the superfluid $\mathrm{BF}$ mixture with the spin-balanced fermion components in the unitarity regime, where the $s$-wave scattering length, $a_{\mathrm{F}}$, which accounts for the interaction between the spin-up and spin-down fermions, is extremely large (ideally, $a_{\mathrm{F}} \rightarrow \pm \infty$ ). Actually, essentially the same Lagrangian (4) also applies to the BF mixture with the fermionic component kept in the BCS regime, when $a_{\mathrm{F}}$ is negative and small (ideally, $a_{\mathrm{F}} \rightarrow-0$ ). In this regime, one is dealing with practically a gas of ideal fermions, because the respective superfluid energy gap is exponentially small. In practical terms, to study the system whose Fermi component falls into the BCS regime, it is sufficient to set $\xi=1$ instead of $\xi=0.45$ in Eq. (4) [7]. Obviously, this change of $\xi$ makes the fermions effectively more repulsive [see Eq. (15)], as the Pauli repulsion attains its maximum at $\xi=1$. We have verified that as the SSB in the mixture emerges chiefly due to $\mathrm{BF}$ attraction, the increase of the intrinsic repulsion in the Fermionic component corresponding to $\xi=1$ makes the natural $\mathrm{BF}$ attraction in the ${ }^{87} \mathrm{Rb}$ and ${ }^{40} \mathrm{~K}$ mixture insufficient for the appearance of the SSB for the relatively small values of $N_{\mathrm{F}}$ and $N_{\mathrm{B}}$ considered previously. To achieve the transition to asymmetric ground states (for the same value of $a_{\perp}=1 \mu \mathrm{m}$ as taken before), it is necessary to consider values of $N_{\mathrm{B}}$ and $N_{\mathrm{F}}$ exceeding 1500 (cf. Figs. 3 and 5, which display the phase diagrams of the mixture with the fermionic component in the unitarity regime for $\left.N_{\mathrm{B}}, N_{\mathrm{F}} \leqslant 1000\right)$.

\section{ANALYTICAL APPROACH: THE THOMAS-FERMI APPROXIMATION FOR THE BOSE COMPONENT}

\section{A. General case}

For the application of the analytical approach, we use the stationary version of general equations (18) and (19), obtained by the substitution of $\phi_{\mathrm{B}, \mathrm{F}}(x, \tau)=\exp \left(-i \mu_{\mathrm{B}, \mathrm{F}} \tau\right) u_{\mathrm{B}, \mathrm{F}}(x)$, where chemical potentials for the localized states must be nonpositive, $\mu_{\mathrm{B}, \mathrm{F}} \leqslant 0$, and real functions $u_{\mathrm{B}, \mathrm{F}}$ obey the following equations (with the prime standing for $d / d x$ ):

$$
\begin{aligned}
& -\frac{1}{2} u_{\mathrm{B}}^{\prime \prime}+\left[-\mu_{\mathrm{B}}+W_{\mathrm{B}}(x)+g_{\mathrm{B}} u_{\mathrm{B}}^{2}+g_{\mathrm{BF}} N_{\mathrm{F}} u_{\mathrm{F}}^{2}\right] u_{\mathrm{B}}=0, \\
& -\frac{1}{8} u_{\mathrm{F}}^{\prime \prime}+\left[-\mu_{\mathrm{F}}+g_{\mathrm{F}} u_{\mathrm{F}}^{4 / 3}+g_{\mathrm{BF}} N_{\mathrm{B}} u_{\mathrm{B}}^{2}+W_{\mathrm{F}}(x)\right] u_{\mathrm{F}}=0 .
\end{aligned}
$$

An essential simplification of Eqs. (26) and (27) can be achieved if the TF approximation may be applied to the former equation, that is, the term with the second derivative may be neglected in it $[3,4,6]$. In the present setting, with the characteristic size of the trapped states $\Delta x \sim 1$ [see Eqs. (23) and Figs. 1 and 6] and the wave functions subject to normalization conditions (21) [hence the amplitudes of normalized density $u_{\mathrm{B}}^{2}(x)$ and $u_{\mathrm{F}}^{2}(x)$ are also $\sim 1$ ], a straightforward consideration of Eq. (26) demonstrates that the kinetic energy (the second derivative) is negligible in comparison with either nonlinear term under conditions $N_{\mathrm{B}} \gg a_{\perp} / a_{\mathrm{B}}$ or $N_{\mathrm{F}} \gg a_{\perp} /\left(10\left|a_{\mathrm{BF}}\right|\right)$. For the value of $a_{\perp}=1 \mu \mathrm{m}$ adopted previously and the values of $a_{\mathrm{B}}$ and $\left|a_{\mathrm{BF}}\right|$ for the ${ }^{87} \mathrm{Rb}$ and ${ }^{40} \mathrm{~K}$ mixture, these conditions reduce to quite realistic inequalities, $N_{\mathrm{B}} \gg 200, N_{\mathrm{F}} \gg 10$. 
If the TF approximation is valid, it allows one to solve Eq. (26) in the following form:

$u_{\mathrm{B}}^{2}(x)=\left\{\begin{array}{lr}g_{\mathrm{B}}^{-1}\left[\left|g_{\mathrm{BF}}\right| N_{\mathrm{F}} u_{\mathrm{F}}^{2}(x)-W_{\mathrm{B}}(x)+\mu_{\mathrm{B}}\right], & \text { at }|x|<x_{0}, \\ 0, & \text { at }|x|>x_{0},\end{array}\right.$

where it is taken into account that we are dealing with $g_{\mathrm{BF}}<0$ and $x_{0}$ is a positive root of equation

$$
u_{\mathrm{F}}^{2}\left(x_{0}\right)=\left(\left|g_{\mathrm{BF}}\right| N_{\mathrm{F}}\right)^{-1}\left[W_{B}\left(x_{0}\right)-\mu_{\mathrm{B}}\right] .
$$

Note that the bosonic chemical potential, $\mu_{\mathrm{B}}$, is not an arbitrary parameter; instead, it must be found from the normalization condition (21), applied to expression (28):

$$
2 \int_{0}^{x_{0}}\left[\left|g_{\mathrm{BF}}\right| N_{\mathrm{F}} u_{\mathrm{F}}^{2}(x)-W_{B}(x)+\mu_{\mathrm{B}}\right] d x=g_{B} .
$$

\section{B. Tractable example}

The substitution of approximation (28) for $u_{\mathrm{B}}^{2}$ into equation (27) for the fermionic function, $u_{\mathrm{F}}(x)$, allows one to reduce the underlying system to a single equation for $u_{\mathrm{F}}(x)$; however, in the general case this equation is quite complex. In particular, the additional equation (29) for $x_{0}$ actually makes the resulting equation for $u_{\mathrm{F}}$ nonlocal. Thus, in the general case, the TF approximation does not yield an explicit analytical solution. Nevertheless, it can be obtained in a special case, when $W_{\mathrm{B}}=0$ [cf. Eq. (22)] and $\mu_{\mathrm{B}}=0$. In this case, Eq. (28) yields a simple local relation,

$$
u_{\mathrm{B}}^{2}(x)=\left(\left|g_{\mathrm{BF}}\right| N_{\mathrm{F}} / g_{\mathrm{B}}\right) u_{\mathrm{F}}^{2}(x),
$$

which is valid at all $x$, and Eq. (30) reduces to a special relation between the boson and fermion numbers,

$$
N_{\mathrm{F}}=g_{\mathrm{B}} /\left|g_{\mathrm{BF}}\right| \equiv-\left(a_{\mathrm{B}} / 3 a_{\mathrm{BF}}\right) N_{\mathrm{B}},
$$

where we have made use of Eqs. (20); note that this approximation is meaningful only in the case when the signs of $a_{\mathrm{B}}$ and $a_{\mathrm{BF}}$ are opposite. For the parameters of the ${ }^{87} \mathrm{Rb}$ and

${ }^{40} \mathrm{~K}$ mixture, Eq. (32) amounts to $N_{\mathrm{F}} \approx 0.127 N_{\mathrm{B}}$. Finally, the single equation for the fermionic stationary function takes the following form, upon the substitution of expression (31):

$$
\begin{aligned}
&- \frac{1}{8} u_{\mathrm{F}}^{\prime \prime}+\left[W_{\mathrm{F}}(x)-\mu_{\mathrm{F}}\right] u_{\mathrm{F}}+\left(3 \pi^{2}\right)^{2 / 3} \frac{3 \xi}{5} N_{\mathrm{F}}^{2 / 3} u_{\mathrm{F}}^{7 / 3} \\
&-\frac{18 a_{\mathrm{BF}}^{2}}{a_{\mathrm{B}} a_{\perp}} N_{\mathrm{F}} u_{\mathrm{F}}^{3}=0,
\end{aligned}
$$

where we have again used Eqs. (20). Note that the last term in Eq. (33) directly illustrates the possibility proposed in this work, namely, that the interaction mediated by the boson field may give rise to an effective attraction in the Fermi component of the BF superfluid mixture: indeed, the coefficient in front of this term is proportional to the square of the scattering length, $a_{\mathrm{BF}}^{2}$, which accounts for the $\mathrm{BF}$ attraction. It is also worth noting that, in the present simplest approximation, which makes it possible to eliminate the boson field and reduce the model to the single equation for the fermionic function, the attractive character of the resulting boson-mediated interaction requires $a_{\mathrm{B}}>0$, that is, the repulsive character of the direct interaction between the bosons. Note that in the case when both the $\mathrm{BF}$ and boson-boson interactions are attractive (i.e., both $a_{\mathrm{B}}$ and $a_{\mathrm{BF}}$ are negative), the present approximation is impossible, according to Eq. (32); in the case of the $\mathrm{BF}$ repulsion and boson-boson attraction (i.e., $a_{\mathrm{B}}<0$ and $a_{\mathrm{BF}}>0$ ), the approximation is possible, but it leads to an effective boson-mediated repulsion between the fermions.

Equation (33) is a variant of the stationary NLSE with two competing nonlinear terms, the self-repulsive one, $\sim N_{\mathrm{F}}^{2 / 3} u_{\mathrm{F}}^{7 / 3}$, and the self-attractive cubic term. For $a_{\perp}=1 \mu \mathrm{m}$ and the scattering lengths corresponding to the ${ }^{87} \mathrm{Rb}$ and ${ }^{40} \mathrm{~K}$ mixture, the coefficient in front of the cubic term is $18 a_{\mathrm{BF}}^{2} /\left(a_{\mathrm{B}} a_{\perp}\right) \approx$ 0.71 , while with $\xi=0.4$ (recall it corresponds to the unitarity regime for the fermion component), the coefficient in front of the repulsive term is $\left(3 \pi^{2}\right)^{2 / 3}(3 \xi / 5) \approx 2.30$.

The SSB controlled by competing nonlinearities (viz., selffocusing cubic and self-defocusing quintic terms) was studied in Refs. [12], where it was concluded that the respective SSB diagrams, showing the asymmetry versus the total norm of the mode (cf. Fig. 2), tend to form a closed loop connecting an initial symmetry-breaking bifurcation and a final symmetryrestoring one. The difference of Eq. (33) is that here the selffocusing (cubic) term has a higher nonlinearity power than its self-defocusing counterpart of power $7 / 3$, and therefore no closed-loop bifurcation diagram is expected in the present case.

The substitution of $u_{\mathrm{F}}(x) \equiv v_{\mathrm{F}} / \sqrt{N_{\mathrm{F}}}$ casts Eq. (33) in a parameter-free form [the respective equation for $v_{\mathrm{F}}$ seems as Eq. (33) with $N_{\mathrm{F}}$ replaced by 1]; of course, this substitution changes normalization (21) for the fermionic function, as the norm of $v_{\mathrm{F}}$ is exactly $N_{\mathrm{F}}$ but not 1 . Thus, in the present approximation, the $\mathrm{BF}$ mixture is described by the universal equation. This circumstance, along with condition (32) necessary for the applicability of Eq. (33), may explain the fact that the border of the trapped states in Fig. 3 is practically a straight line with the slope equal to 1 , which runs into the SSB border at a critical value of $N_{\mathrm{F}}$ [the latter one actually corresponds to the critical norm of field $v_{\mathrm{F}}(x)$ at which the SSB occurs in the framework of Eq. (33)].

The actual SSB point generated by Eq. (33) can be predicted in an approximate analytical form by means of the two-mode expansion, which, as mentioned previously, is commonly used for the description of SSB effects in DWP settings [16], assuming that the stationary solution is approximated by a superposition of two linear modes, $u_{ \pm}(x)$, which are trapped in the left and right potential wells:

$$
u_{\mathrm{F}}(x)=A_{+} u_{+}(x)+A_{-} u_{-}(x) .
$$

Symmetric states correspond to $A_{+}=A_{-} \equiv A_{0}$ in Eq. (34). The SSB border can be found by looking for a point where a solution with an infinitely small antisymmetric perturbation $(\delta)$, $A_{ \pm}=A_{0} \pm \delta$, branches off from the parent symmetric state. By performing this analysis (we do not display straightforward details here), one finds that the value of $A_{0}$ for SSB scales inversely proportional to $\sqrt{N_{\mathrm{F}}}$, and hence the increase of the number of particles facilitates the transition to the asymmetric ground state, as one may expect.

\section{CONCLUSION}

The objective of this work is to extend the analysis of the SSB in DWP settings, which was recently studied in BEC 
and bosonic mixtures, to the BF mixtures. The system is described by the GPE for the bosons, which is nonlinearly coupled to the equation for the fermionic order parameter derived from the density functional in the unitarity limit (in fact, a similar model also applies to the BF mixture with the fermionic component kept in the BCS regime). Direct symmetry breaking in the Fermi superfluid trapped in the DWP is impossible, as it must be induced by attractive interactions, while density perturbations in the degenerate fermionic gas interact repulsively. Nevertheless, we have demonstrated that the SSB is possible in the mixture of ${ }^{87} \mathrm{Rb}$ and ${ }^{40} \mathrm{~K}$ atoms, due to the attraction between fermions and bosons. The most interesting case, when the effective SSB in the fermionic component could be studied in the pure form, is that when the fermions are subject to the action of the DWP, there is no potential confining the bosons. We have also investigated the alternative situation, with the DWP acting solely on the bosons. Our phase diagrams in the $\left(N_{\mathrm{F}}, N_{\mathrm{B}}\right)$ plane, produced by means of numerical methods, clearly show that the interatomic attractions can produce both symmetric and symmetry-broken localization of the atoms which are not subject to the direct action of the trapping potential. By applying the TF aproximation to the bosonic equation, we also developed an analytical approximation, which allows us to reduce the model to the single equation for the fermionic function. In the latter case, the model explicitly demonstrates the generation of the effective attraction between fermions mediated by the bosons.

The analysis reported in this work can be extended in other directions. A straightforward generalization may deal with the system including the confining potential in both components, as well as a more general analysis of the TF approximation. A challenging possibility is to predict similar effects in multidimensional BF mixtures.

\section{ACKNOWLEDGMENTS}

The work of SKA was partially supported by Conselho Nacional de Desenvolvimento Científico e Tecnológico and Fundação de Amparo à Pesquisa do Estado de São Paulo (Brazil). BAM acknowledges the hospitality of the Department of Physics "Galileo Galilei" at the University of Padua, Italy, and support from the German-Israel Foundation through Grant No. $149 / 2006$.
[1] M. H. Anderson, J. R. Ensher, M. R. Matthews, C. E. Wieman, and E. A. Cornell, Science 269, 198 (1995); C. C. Bradley, C. A. Sackett, J. J. Tollett, and R. G. Hulet, Phys. Rev. Lett. 75, 1687 (1995); K. B. Davis, M.-O. Mewes, M. R. Andrews, N. J. van Druten, D. S. Durfee, D. M. Kurn, and W. Ketterle, ibid. 75, 3969 (1995).

[2] B. DeMarco and D. Jin, Science 285, 1703 (1999).

[3] F. Dalfovo, S. Giorgini, L. P. Pitaevskii, and S. Stringari, Rev. Mod. Phys. 71, 463 (1999).

[4] L. P. Pitaevskii and S. Stringari, Bose-Einstein Condensation (Clarendon Press, Oxford, 2003).

[5] S. Giorgini, L. P. Pitaevskii, and S. Stringari, Rev. Mod. Phys. 80, 1215 (2008); H. T. C. Stoof, K. B. Gubbels, and D. B. M. Dickrsheid, Ultracold Quantum Fields (Springer, Dordrecht, 2009).

[6] E. Lipparini, Modern Many-Particle Physics: Atomic Gases, Nanostructures, and Quantum Liquids (World Scientific, Singapore, 2006).

[7] N. Manini and L. Salasnich, Phys. Rev. A 71, 033625 (2005); G. Diana, N. Manini, and L. Salasnich, ibid. 73, 065601 (2006).

[8] L. Salasnich, N. Manini, and F. Toigo, Phys. Rev. A 77, 043609 (2008); F. Ancilotto, L. Salasnich, and F. Toigo, ibid. 79, 033627 (2009); L. Salasnich and F. Toigo, ibid. 78, 053626 (2008); L. Salasnich, F. Ancilotto, and F. Toigo, Laser Phys. Lett. 7, 78 (2010).

[9] S. K. Adhikari and L. Salasnich, Phys. Rev. A 78, 043616 (2008); New J. Phys. 11, 023011 (2009); S. K. Adhikari, Laser Phys. Lett. 6, 901 (2009); Phys. Rev. A 79, 023611 (2009).

[10] S. K. Adhikari and B. A. Malomed, Phys. Rev. A 74, 053620 (2006); Europhys. Lett. 79, 50003 (2007); Physica D 238, 1402 (2009).

[11] G. J. Milburn, J. Corney, E. M. Wright, and D. F. Walls, Phys. Rev. A 55, 4318 (1997); A. Smerzi, S. Fantoni, S. Giovanazzi, and S. R. Shenoy, Phys. Rev. Lett. 79, 4950 (1997); S. Raghavan, A. Smerzi, S. Fantoni, and S. R. Shenoy, Phys. Rev. A 59, 620 (1999); K. W. Mahmud, H. Perry, and W. P. Reinhardt, ibid. 71, 023615 (2005); E. Infeld, P. Zin, J. Gocałek, and M. Trippenbach, Phys. Rev. E 74, 026610 (2006); G. Theocharis, P. G. Kevrekidis, D. J. Frantzeskakis, and P. Schmelcher, ibid. 74, 056608 (2006); G. L. Alfimov and D. A. Zezyulin, Nonlinearity 20, 2075 (2007).

[12] L. Albuch and B. A. Malomed, Mathematics and Computers in Simulation 74, 312 (2007); Z. Birnbaum and B. A. Malomed, Physica D 237, 3252 (2008).

[13] A. W. Snyder, D. J. Mitchell, L. Poladian, D. R. Rowland, and Y. Chen, J. Opt. Soc. Am. B 8, 2102 (1991).

[14] E. B. Davies, Commun. Math. Phys. 64, 191 (1979).

[15] J. C. Eilbeck, P. S. Lomdahl, and A. C. Scott, Physica D 16, 318 (1985).

[16] E. A. Ostrovskaya, Y. S. Kivshar, M. Lisak, B. Hall, F. Cattani, and D. Anderson, Phys. Rev. A 61, 031601(R) (2000); R. D’Agosta, B. A. Malomed, and C. Presilla, Phys. Lett. A 275, 424 (2000); R. K. Jackson and M. I. Weinstein, J. Stat. Phys. 116, 881 (2004); V. S. Shchesnovich, B. A. Malomed, and R. A. Kraenkel, Physica D 188, 213 (2004); D. Ananikian and T. Bergeman, Phys. Rev. A 73, 013604 (2006); C. Wang, P. G. Kevrekidis, N. Whitaker, and B. A. Malomed, Physica D 237, 2922 (2008); E. W. Kirr, P. G. Kevrekidis, E. Shlizerman, and M. I. Weinstein, SIAM J. Math. Anal. 40, 566 (2008).

[17] A. Gubeskys and B. A. Malomed, Phys. Rev. A 75, 063602 (2007); M. Matuszewski, B. A. Malomed, and M. Trippenbach, ibid. 75, 063621 (2007); M. Trippenbach, E. Infeld, J. Gocałek, M. Matuszewski, M. Oberthaler, and B. A. Malomed, ibid. 78, 013603 (2008).

[18] M. Albiez, R. Gati, J. Fölling, S. Hunsmann, M. Cristiani, and M. K. Oberthaler, Phys. Rev. Lett. 95, 010402 (2005); for a review, see R. Gati and M. Oberthaler, J. Phys. B 40, R61 (2007). 
[19] T. Mayteevarunyoo and B. A. Malomed, J. Opt. A: Pure Appl. Opt. 11, 094015 (2009).

[20] C. Wang, G. Theocharis, P. G. Kevrekidis, N. Whitaker, K. J. H. Law, D. J. Frantzeskakis, and B. A. Malomed, Phys. Rev. E 80, 046611 (2009).

[21] B. Liu, L.-B. Fu, S.-P. Yang, and J. Liu, Phys. Rev. A 75, 033601 (2007).

[22] B. Xiong, J. Gong, H. Pu, W. Bao, and B. Li, Phys. Rev. A 79, 013626 (2009).

[23] A. Sacchetti, Phys. Rev. Lett. 103, 194101 (2009).

[24] T. Mayteevarunyoo, B. A. Malomed, and G. Dong, Phys. Rev. A 78, 053601 (2008); C. Wang, P. G. Kevrekidis, N. Whitaker, D. J. Frantzeskakis, S. Middelkamp, and P. Schmelcher, Physica D 238, 1362 (2009).

[25] C. Wang, P. G. Kevrekidis, N. Whitaker, and B. A. Malomed, Physica D 237, 2922 (2008); I. I. Satija, R. Balakrishnan, P. Naudus, J. Heward, M. Edwards, and C. W. Clark, Phys. Rev. A 79, 033616 (2009); W. Wang, J. Phys. Soc. Jpn. 78, 094002 (2009); C. Lee, Phys. Rev. Lett. 102, 070401 (2009).

[26] C. Wang, P. G. Kevrekidis, N. Whitaker, T. J. Alexander, D. J. Frantzeskakis, and P. Schmelcher, J. Phys. A Math. Theor. 42, 035201 (2009); B. Juliá-Diaz, M. Guilleumas, M. Lewenstein, A. Polls, and A. Sanpera, Phys. Rev. A 80, 023616 (2009); B. Juliá-Diaz, M. Mele-Messeguer, M. Guilleumas, and A. Polls, ibid. 80, 043622 (2009).

[27] S. F. Caballero-Benítez, E. A. Ostrovskaya, M. Gulácsí, and Y. S. Kivshar, J. Phys. B 42, 215308 (2009).

[28] S. K. Adhikari, H. Lu, and H. Pu, Phys. Rev. A 80, 063607 (2009).
[29] G. Modugno, G. Roati, F. Riboli, F. Ferlaino, R. J. Brecha, and M. Inguscio, Science 297, 2240 (2002); G. Roati, F. Riboli, G. Modugno, and M. Inguscio, Phys. Rev. Lett. 89, 150403 (2002).

[30] L. Salasnich, Laser Phys. 19, 642 (2009).

[31] L. Salasnich, Laser Phys. 12, 198 (2002); L. Salasnich, A. Parola, and L. Reatto, Phys. Rev. A 65, 043614 (2002).

[32] T. Volz, S. Dürr, S. Ernst, A. Marte, and G. Rempe, Phys. Rev. A 68, 010702 (2003).

[33] K. Mølmer, Phys. Rev. Lett. 80, 1804 (1998); P. Capuzzi, A. Minguzzi, and M. P. Tosi, Phys. Rev. A 67, 053605 (2003); 68, 033605 (2003); W. Yi and L.-M. Duan, Europhys. Lett. 75, 854 (2006); A. M. Belemuk, N. M. Chtchelkatchev, and V. N. Ryzhov, and S.-T. Chui, Phys. Rev. A 73, 053608 (2006); Y. V. Bludov and V. V. Konotop, ibid. 74, 043616 (2006); S. K. Adhikari, ibid. 72053608 (2005); 70, 043617 (2004); New J. Phys. 8, 258 (2006).

[34] S. K. Adhikari and B. A. Malomed, Phys. Rev. A 76, 043626 (2007).

[35] P. Muruganandam and S. K. Adhikari, Comput. Phys. Commun. 180, 1888 (2009).

[36] L. Salasnich and F. Toigo, Phys. Rev. A 75, 013623 (2007); L. Salasnich, S. K. Adhikari, and F. Toigo, ibid. 75, 023616 (2007).

[37] K. E. Strecker, G. B. Partridge, A. G. Truscott, and F. G. Hulet, Nature 417, 150 (2002); L. Khaykovich, F. Schreck, G. Ferrari, T. Bourdel, J. Cubizolles, L. D. Carr, Y. Castin, and C. Salomon, Science 296, 1290 (2002). 